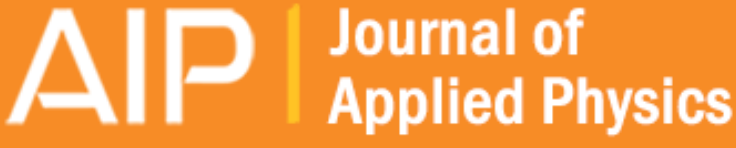

Structural and magnetic properties of planar nanowire arrays of Co grown on oxidized vicinal silicon (111) templates

S. K. Arora, B. J. O’Dowd, C. Nistor, T. Balashov, B. Ballesteros, A. Lodi Rizzini, J. J. Kavich, S. S. Dhesi, P. Gambardella, and I. V. Shvets

Citation: Journal of Applied Physics 111, 07 E342 (2012); doi: 10.1063/1.3679033

View online: http://dx.doi.org/10.1063/1.3679033

View Table of Contents: http://scitation.aip.org/content/aip/journal/jap/111/7?ver=pdfcov

Published by the AIP Publishing

\section{Articles you may be interested in}

Structural and magnetic characterization of as-prepared and annealed FeCoCu nanowire arrays in ordered anodic aluminum oxide templates

J. Appl. Phys. 115, 133904 (2014); 10.1063/1.4870289

Anisotropic magnetic properties of bi-layered structure of ordered Co nanowire array: Micromagnetic simulations and experiments

J. Appl. Phys. 114, 173905 (2013); 10.1063/1.4829034

Template-based synthesis and magnetic properties of $\mathrm{Mn}-\mathrm{Zn}$ ferrite nanotube and nanowire arrays

J. Appl. Phys. 111, 026104 (2012); 10.1063/1.3678580

Influence of an Au capping layer on the magnetic properties of CoPt nanowires

Appl. Phys. Lett. 98, 252507 (2011); 10.1063/1.3601748

Magnetic properties of planar arrays of Fe-nanowires grown on oxidized vicinal silicon (111) templates

J. Appl. Phys. 109, 07B106 (2011); 10.1063/1.3554264

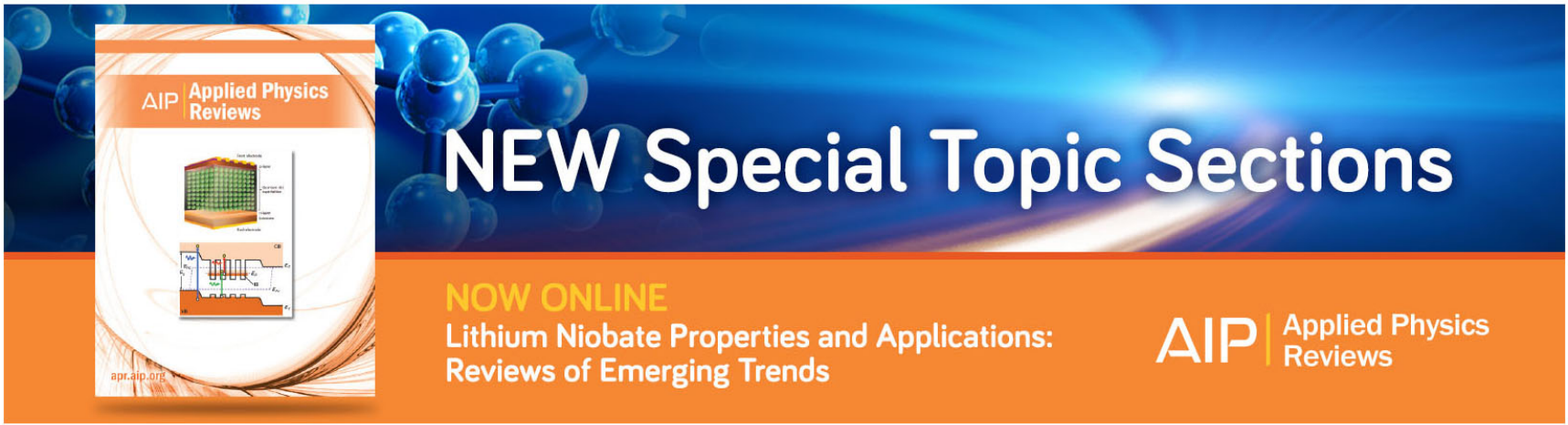




\title{
Structural and magnetic properties of planar nanowire arrays of Co grown on oxidized vicinal silicon (111) templates
}

\author{
S. K. Arora, ${ }^{1, a)}$ B. J. O'Dowd, ${ }^{1}$ C. Nistor, ${ }^{2}$ T. Balashov, ${ }^{2}$ B. Ballesteros, ${ }^{2}$ A. Lodi Rizzini, ${ }^{2}$ \\ J. J. Kavich, ${ }^{2}$ S. S. Dhesi, ${ }^{3}$ P. Gambardella, ${ }^{2,4}$ and I. V. Shvets ${ }^{1}$ \\ ${ }^{1}$ Centre for Research on Adaptive Nanostructures and Nanodevices (CRANN) and School of Physics, \\ Trinity College Dublin, Dublin 2, Ireland \\ ${ }^{2}$ Catalan Institute of Nanotechnology (ICN-CIN2), UAB Campus, E-08193 Barcelona, Spain \\ ${ }^{3}$ Diamond Light Source, Chilton, Didcot, Oxfordshire OX11 ODE, United Kingdom \\ ${ }^{4}$ ICREA and Universitat Autonoma de Barcelona, E-08193 Barcelona, Spain
}

(Presented 2 November 2011; received 10 October 2011; accepted 1 December 2011; published online 9 March 2012)

\begin{abstract}
We fabricated planar arrays of Co nanowires (NWs) on oxidized step-bunched $\mathrm{Si}$ (111) templates using shallow angle deposition. These planar NW arrays exhibit ferromagnetic behavior at room temperature for NW widths down to $25 \mathrm{~nm}$. The NWs possess polycrystalline character with hcp-crystal structure, and present a lightly oxidized interface when capped with $\mathrm{MgO}$. The magnetic anisotropy of the NW array is dominated by the shape anisotropy, which keeps the magnetization in-plane with easy axis parallel to the wires. By reducing the inter-wire separation, we obtain NW arrays with reduced coercivity demonstrating the importance of magneto-static interactions in determining the magnetic properties of the NWs. (C) 2012 American Institute of Physics. [doi:10.1063/1.3679033]
\end{abstract}

Ordered arrays of nanowires (NWs) have received considerable attention due to their promising application potential in spin electronic devices and novel physics perspective. ${ }^{1-3}$ Bottom up self-assembly methods have been used to fabricate magnetic NW arrays of wire width ranging from single atom to several hundred $\mathrm{nm}$ are reported on a variety of templates. ${ }^{1-8}$ Control over the size distribution of magnetic NWs has been demonstrated in carefully prepared porous anodized aluminum oxide (AAO) templates. ${ }^{4,5}$ In AAO templates NW growth occurs perpendicular to the template surface. Planar NW arrays on self-assembled templates could be formed using step flow, step decoration ${ }^{6-9}$ and reactive deposition epitaxy ${ }^{10}$ on vicinal templates. The planar NW arrays produced using these methods exhibit superparamagnetic (SPM) behavior at room temperature (RT) due to small thickness (a few monolayer) of NWs. Moreover, these processes are material selective in nature, which restricts their utility for application purposes. It has been demonstrated that by using shallow angle deposition one can overcome SPM as it enables controlled growth of magnetic nanostructures that are thick enough to exhibit ferromagnetism at room temperature. ${ }^{11,12}$ We make use of the atomic terrace low angle shadowing (ATLAS) technique ${ }^{12,13}$ to grow several nm thick Co-NW arrays on oxidized step-bunched Si templates. Here, we report on the structural and magnetic properties of planar NW arrays of Co with varying wire widths $(25-70 \mathrm{~nm})$ grown on oxidized step bunched Si surfaces by means of the ATLAS method.

We grew two sets of Co NW array samples on stepbunched vicinal $\mathrm{Si}$ (111) templates of $110 \mathrm{~nm}$ periodicity (sum of terrace width $(85 \mathrm{~nm})$ and step bunch width of $(25$ $\mathrm{nm})$ ) were prepared under identical conditions using a dc-

\footnotetext{
a) Author to whom correspondence should be addressed. Electronic mail: aroras@tcd.ie.
}

current annealing. ${ }^{13,14}$ For topographical investigations we prepared Co-NW arrays without cap layer, whereas for magnetization and transmission electron microscopy (TEM) studies, the arrays were capped with a $5 \mathrm{~nm} \mathrm{MgO}$ layer. The templates were oxidized at $830^{\circ} \mathrm{C}$ for a duration of $15 \mathrm{~h}$ producing a $110 \mathrm{~nm}$ thick amorphous surface oxide layer.

The Co-NW arrays were grown at RT onto the oxidized templates using the ATLAS method. Details of the ATLAS method are given elsewhere. ${ }^{12,13}$ The topography characterization of the templates and NW arrays was performed using a scanning electron microscope (SEM, Zeiss Ultra) and atomic force microscope (AFM Solver Pro, NT MDT). For structural studies we used a high resolution transmission electron microscope (HRTEM, FEI Tecnai F30) operated at 300 $\mathrm{kV}$. Sample cross-sections for HRTEM observation were prepared using focused ion beam(FIB) on a Helios Nanolab microscope. The magnetic properties of the NW arrays were examined using a vibrating sample magnetometer (Quantum Design - Physical Property Measurements System) with a sensitivity of $5 \times 10^{-7} \mathrm{emu}$. The diamagnetic contribution from the substrate was subtracted from the measured data by subtracting a $M-H$ loop of the substrate of similar dimensions. Element specific $\mathrm{x}$-ray absorption (XAS) and x-ray magnetic circular dichroism (XMCD) experiments were carried out in total electron yield at the I06 beamline of the Diamond synchrotron facility.

Figure 1 shows the SEM images of the two uncapped Co NW array samples of $25 \mathrm{~nm}$ (Fig. 1(a)) and $70 \mathrm{~nm}$ (Fig. 1(b)) wire widths grown on $110 \mathrm{~nm}$ periodicity oxidized Si (111) templates. Thickness of the NW arrays found from the AFM height images and corresponding height profile analysis (not shown) were found to be 3 and $1.4 \mathrm{~nm}$, respectively. It is clear from the figure that the NW arrays are well separated and quite regular. From the image (Fig. 1(b)) one can clearly see 

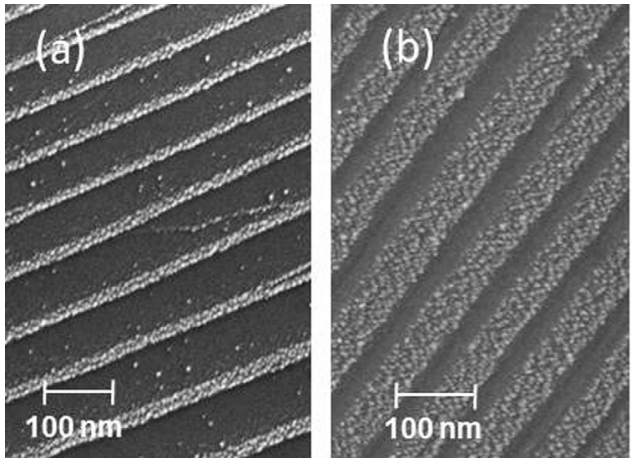

FIG. 1. (a) SEM image of an uncapped Co-NW array ( $3 \mathrm{~nm}$ thick) grown with the deposition flux directed uphill (deposition angle of $3^{\circ}$ ) on a $110 \mathrm{~nm}$ oxidized Si (111) vicinal template. (b) SEM image of a small thickness (1.2 $\mathrm{nm}) \mathrm{Co}-\mathrm{NW}$ array on $110 \mathrm{~nm}$ template with the deposition flux directed downhill (deposition angle of $2.5^{\circ}$ ) illustrating the presence of isolated 3D islands of Co during the initial stages of growth. Direction of deposition flux in images is from left to right.

that the shadowing caused by the step-bunches leads to partial coverage of the terraces $(\sim 80 \%)$ producing NW arrays with average wire width of $70 \mathrm{~nm}$. Another noticeable feature visible is the island type morphology of the Co NWs. In the initial stages of growth, discontinuous chains of aligned Co islands form on the terraces. With increasing thickness, the density of the islands increases, eventually leading to the formation of a nanowire of coalesced islands. We did not observe any noticeable change in the NW morphology for NW thicknesses greater than $3 \mathrm{~nm}$.

We performed magnetization studies on two $\mathrm{MgO}(5 \mathrm{~nm}$ thick) capped Co-NW arrays grown on $110 \mathrm{~nm}$ periodicity oxidized step-bunched $\mathrm{Si}$ (111) templates with $4.5 \mathrm{~nm}$ nominal thickness and wire widths of 70 and $25 \mathrm{~nm}$. We refer to them as samples 1 and 2 hereafter. Figure 2 shows the crosssectional TEM images of sample 1. The width of the NWs was found to vary between 50 and $70 \mathrm{~nm}$ for this particular sample (Fig. 2(a)), which is related to the statistical fluctuations in the periodicity of the self-assembled template. From the analysis of the TEM images we infer that the majority of the Co-NWs appear to be polycrystalline (see Fig. 2(b)), as expected for nanowires formed of coalesced islands. In some cases the structure of single crystallites can be determined, and it is found to be mostly $h c p$-Co. A HRTEM image of a $h c p-\mathrm{Co}$ crystallite viewed along the [001]zone axis and its correspond-
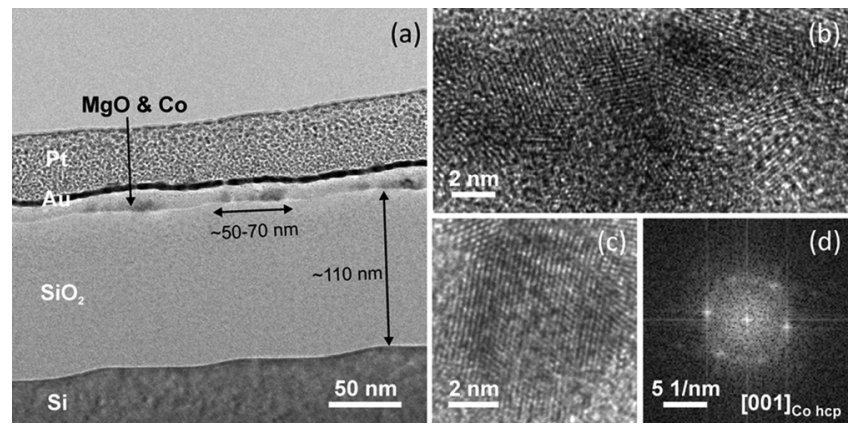

FIG. 2. (a) Cross-sectional TEM image of a $5 \mathrm{~nm}$ thick Co-NW array on a $110 \mathrm{~nm}$ average periodicity oxidized Si template (sample 1). (b) Highresolution TEM image showing the polycrystalline nature of the Co-NWs. (c) HRTEM image of a Co-NW crystallite with hcp-Co structure viewed along the [001] zone axis and (d) its corresponding fast Fourier transform.

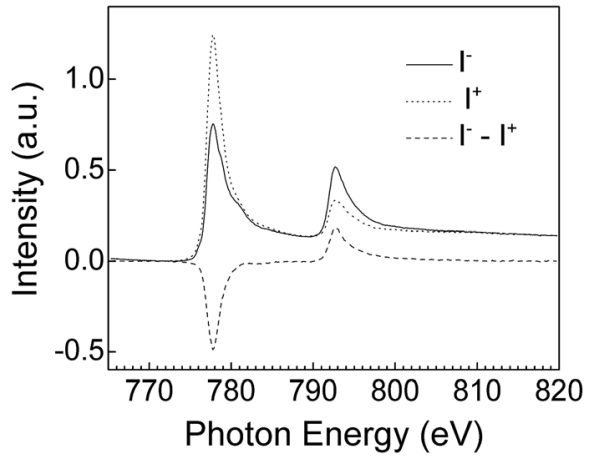

FIG. 3. X-ray absorption spectra recorded at the $L_{3,2}$ edge of Co for an array of NWs with average width $70 \mathrm{~nm}$ grown on an oxidized silicon template with $110 \mathrm{~nm}$ periodicity (sample 1). The spectra were recorded for magnetization parallel and antiparallel to the x-ray helicity (dotted and solid curves, respectively) at an applied field of $1.95 \mathrm{~T}$. The corresponding XMCD $\left(\mathrm{I}^{-}-\mathrm{I}^{+}\right)$signal is shown with the dashed line curve.

ing fast Fourier transform (FFT) are shown in Figs. 2(c) and 2(d), respectively. In a few areas we also observed $f c c$-Co and $f c c-\mathrm{CoO}$ structures, where oxidation is likely due to the proximity of the $\mathrm{MgO}$ cap layer.

Figure 3 shows the $\mathrm{X}$-ray absorption and XMCD spectra spectra of sample 1 taken at the $L_{2,3}$ edges in total electron yield mode using circularly polarized light with $99 \pm 1 \%$ polarization. The XAS spectra taken with negative and positive circular polarization ( $\mathrm{I}^{-}$and $\mathrm{I}^{+}$, respectively) were normalized to the incident photon flux.

The XAS line shape is typical of Co metal, with only very mild signs of oxidation as seen in the shoulder $1 \mathrm{eV}$ above the $\mathrm{Co} L_{3}$ edge peak at $777.7 \mathrm{eV}$ and a faint pre-edge feature at $776 \mathrm{eV}$. This indicates that most of the wires are metallic, even when covered with $\mathrm{MgO}$. The magnitude of the XMCD signal, $\left(\mathrm{I}^{-}-\mathrm{I}^{+}\right)$, compared to the average XAS, $\left(\mathrm{I}^{-}+\mathrm{I}^{+}\right) / 2$, confirms the presence of a sizable magnetic moment. From the sum rule analysis we extract the spin and orbital magnetic moments per Co atom. ${ }^{15,16} \mathrm{We}$ estimate that the average spin magnetic moment of the nanowires amounts to $1.4 \pm 0.1 \mu_{\mathrm{B}}$ /atom. Whereas value of the orbital magnetic moment is $0.13 \pm 0.02 \mu_{\mathrm{B}} /$ atom. These values are about $10 \%$ smaller compared to those reported for bulk hcp $\mathrm{Co},{ }^{15}$ probably due to the small fraction of oxidized Co in the wires. Note that due to the relatively large thickness of the wires, dimensionality effects do not play a role here. ${ }^{17}$

Figure 4 shows the magnetization hysteresis $(\mathrm{M}-\mathrm{H})$ loops of both samples measured at $300 \mathrm{~K}$ with an in-plane applied field directed either along $\left(\mathrm{H}_{\|}\right)$or across $\left(\mathrm{H}_{\perp}\right)$ the
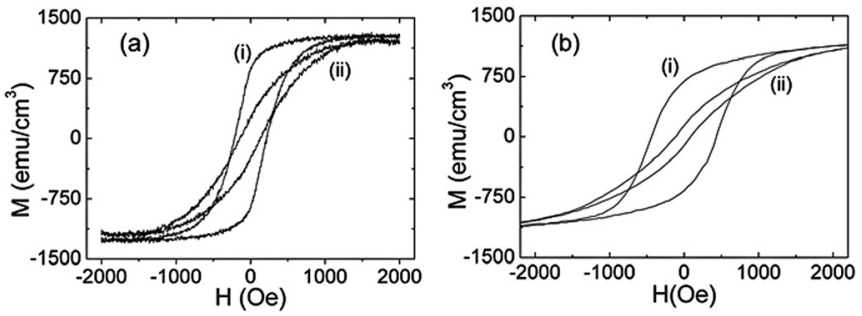

FIG. 4. Magnetization hysteresis loops measured at $300 \mathrm{~K}$ with an in-plane magnetic field directed either along $\left(\mathrm{H}_{\|}\right.$, curves labelled (i)) or across $\left(\mathrm{H}_{\perp}\right.$, curves labelled (ii)) the length of the NWs for (a) sample 1 and (b) sample 2. 
wires (step-edges). Values of coercivity, $\mathrm{H}_{\mathrm{C}}$ for $\mathrm{H}_{\|}\left(\mathrm{H}_{\perp}\right)$ are found to be 215 (140) Oe and 420(130) Oe for sample 1 and sample 2, respectively. Corresponding values of the remanence $\left(\mathrm{M}_{\mathrm{R}}\right)$ are $60(20) \%$ and $54(18) \%$, respectively (the values of $M_{R}$ quoted here are within $2 \%$ ). The $M_{R}$ in our measurements is defined as the ratio of the magnetization at zero and $10 \mathrm{kOe}$ magnetic fields $\left(\mathrm{M}_{\mathrm{s}}(0) / \mathrm{M}_{\mathrm{s}}(10\right.$ $\mathrm{kOe})(\times 100)$. Magnitude of $\mathrm{H}_{\mathrm{C}}$ is found to be greater for sample 2 as compared to sample 1 while the values of $M_{R}$ for both samples are comparable. As expected for wires with large shape anisotropy, the $\mathrm{M}-\mathrm{H}$ loops are square for the field applied parallel to the wires $\left(\mathrm{H}_{\|}\right)$, whereas for $\mathrm{H}_{\perp}$ the loops are sheared. One also notices that the $\mathrm{H}_{\mathrm{C}}$ of the planar $\mathrm{NW}$ arrays is strongly enhanced as compared to the measured $\mathrm{H}_{\mathrm{C}}$ ( $\sim 10 \mathrm{Oe})$ of a $5 \mathrm{~nm}$ continuous Co-film grown at normal incidence on a flat oxidized Si substate. It is clear from the magnetization data that the shape of the hysteresis loops deviates from the expected perfectly square loop for the $\mathrm{H}_{\|}$ case and linear closed loop for the $\mathrm{H}_{\perp}$ case.

Both samples exhibit an easier approach toward magnetic saturation (we refer to points where loop closes and the initial susceptibility) for $\mathrm{H}_{\|}$compared to $\mathrm{H}_{\perp}$, indicating that the magnetic easy axis is along the length of the wires, which suggests that the effective magnetic anisotropy $\left(\mathrm{K}_{\mathrm{eff}}\right)$ is dominated by the shape anisotropy (KS) owing to the large aspect ratio of the wires. We further consider the effect of other contributions to $\mathrm{K}_{\mathrm{eff}}$. Because of polycrystalline nature of the NWs, contribution from magnetocrystalline anisotropy (for fcc- and hcp-Co phases magnitude of $\mathrm{K}_{1}$ is $6.1 \times 10^{5}$ and $5 \times 10^{6} \mathrm{erg} / \mathrm{cm}^{3}$ respectively) expected to be an order of magnitude smaller than the estimated shape anisotropy energy density, $\mathrm{K}_{\mathrm{s}}=\pi \mathrm{M}_{\mathrm{s}}^{2}\left(6.0 \times 10^{6} \mathrm{erg} / \mathrm{cm}^{3}\right)$ for large aspect ratio Co NWs. From temperature dependent magnetization studies we find that the the shape related uniaxial anisotropy is preserved down to $10 \mathrm{~K}$.

In order to understand the observed differences in $\mathrm{H}_{\mathrm{C}}$ on the applied field direction and width of the wires, we need to consider the influence of long range dipolar interactions on the magnetic anisotropy. Magnetostatic interactions depends on the direction of field, length (L) to width (d) ratio, and inter-wire separation D. ${ }^{18,19}$ For the case of large aspect ratio nanowires $(\mathrm{d} / \mathrm{L} \ll 1)$ which are homogeneously magnetized, the increase of magnetostatic interaction results in the magnetization reversal of some nanowires. Assuming that the reversal of an individual nanowire produces a decrease of magnetostatic energy $E_{v}$ that equals to the magnetic anisotropy barrier $\Delta E$, the macroscopic coercivity can be written as ${ }^{19}$

$$
H c=\frac{2 K}{\mu_{0} M_{s}}\left[1-\left(\frac{N E_{v}}{K}\right)^{1 / 2}\right]
$$

where $2 \mathrm{~K} / \mu_{0} \mathrm{M}_{\mathrm{S}}$ denotes the intrinsic $\mathrm{Hc}$ due to magnetic ansitropies, $\mathrm{K}$. This interaction energy $E_{V}$, including multi- polar components is found to depend quadratically on the ratio, $r=d / D .{ }^{19} H_{\perp}$ Our observation of enhanced $H_{C}$ with the decrease in $d / D$ is in agreement with the prediction of this model. We find that for closely spaced NW arrays (sample 1 with $\mathrm{r}=1.75) \mathrm{H}_{\mathrm{C}}$ is lower compared to the case of noninteracting wires with greater inter-wire separation (sample 2 with $r=0.36$ ). We also observe that for both samples $\mathrm{H}_{\mathrm{C} \|}>\mathrm{H}_{\mathrm{C} \perp}$, which is fully consistent with the shape anisotropy origin of the enhanced $\mathrm{H}_{\mathrm{C}}$ as discussed qualitatively within the Stoner-Wohlfarth model. ${ }^{4}$ A comparable value of $M_{R}$ for both the samples could be attributed to the size effects. In the absence of size effects one would have expected a lower value of $\mathrm{M}_{\mathrm{R}}$ for sample 1 as compared to sample 2 .

In summary, we have shown that ATLAS grown planar NW arrays of Co with widths down to $25 \mathrm{~nm}$ are ferromagnetic at RT and exhibit in-plane uniaxial anisotropy with easy axis along the length of the wires. The magnetic anisotropy of the NW arrays is dominated by their shape, which leads to an enhanced $\mathrm{H}_{\mathrm{C}}$ and longitudinal easy axis. Whereas for reduced inter-wire separation and larger wire width the contribution from dipolar interactions is found to influence the magnetic properties leading to a reduced $\mathrm{H}_{\mathrm{C}}$.

Work supported by the Science Foundation Ireland (SFI) under Contract No. 06/IN.1/I91, ERA-Net NANOWAVE program, the Irish Government's Program (ISPIRE) for Research in Third Level Institutions, Cycle 4, National Development Plan 2007-2013, the Spanish Ministerio de Ciencia y Innovación (Grant Nos. EUI2008-03884 and PTA2008-1108-I), Agència de Gestió d'Ajuts Universitaris i de Recerca (Grant No. 2009 SGR 695), and Nanoaracat.

${ }^{1}$ C. Teichert, Appl. Phys. A 76, 653 (2003).

${ }^{2}$ P. Schio et al., Phys. Rev. B 82, 094436 (2010).

${ }^{3}$ C. Carbone et al., Adv. Funct. Mater. 21, 1212 (2011).

${ }^{4}$ J. Sanchez-Barriga et al., Phys. Rev. B 80, 184424 (2009).

${ }^{5}$ S. D. Col, M. Darques, O. Fruchart, and L. Cagnon, Appl. Phys. Lett. 98, 112501 (2011).

${ }^{6}$ P. Gambardella et al., Phys. Rev. Lett. 93, 077203 (2004).

${ }^{7}$ Ruihua Cheng et al., Phys. Rev. B 72, 014409 (2005).

${ }^{8}$ H. J. Elmers et al., Phys. Rev. Lett. 73, 898 (1994).

${ }^{9}$ P. O. Jubert, O. Fruchart, and C. Meyer, J. Magn. Magn. Mater. 242, 565 (2002).

${ }^{10}$ K. Radican, N. Berdunov, and I. V. Shvets, Phys. Rev. B 77, 085417 (2008).

${ }^{11}$ J. Oster et al., J. Appl. Phys. 97, 014303 (2005).

${ }^{12}$ F. Cuccureddu et al., Rev. Sci. Instrum. 79, 053907 (2008).

${ }^{13}$ S. K. Arora et al., J. Appl. Phys. 109, 07B106 (2011).

${ }^{14}$ B. J. Gibbons, J. Noffsinger, and P. Pelz, Surf. Sci. Lett. 575, L51 (2005).

${ }^{15}$ C. T. Chen et al., Phys. Rev. Lett. 75, 152 (1995).

${ }^{16}$ P. Carra, B. T. Thole, M. Altarelli, and X. Wang, Phys. Rev. Lett. 70, 694 (1993).

${ }^{17} \mathrm{P}$. Gambardella et al., Nature 416, 301 (2002).

${ }^{18}$ F. Zighem, T. Maurer, F. Ott, and G. Chaboussant, J. Appl. Phys. 109, 013910 (2011).

${ }^{19}$ M. Vazquez et al., J. Appl. Phys. 95, 6642 (2011). 